\title{
Improving Time-Dependent Deformation Measurement
}

\section{Cuiru Sun}

Ryerson University

\section{Jinlong Chen}

Tianjin University

\section{Hua Lu}

Ryerson University

Sun, C., Chen, J., \& Lu, H. (2008, September 1). Improving time-dependent deformation measurement. SPIE.

doi:10.1117/2.1200809.1243

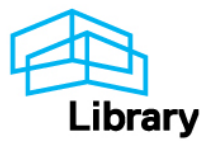


$10.1117 / 2.1200809 .1243$

\section{Improving time-dependent deformation measurement}

\section{Cuiru Sun, Jinlong Chen, and Hua Lu}

A new method for characterizing shape changes over time may improve nondestructive testing.

Phase-shifted digital speckle shearography (PSDSS) is a technique that uses the interference patterns of light waves to measure the slope of a deformed surface. ${ }^{1}$ It shows promise for nondestructive testing of aircraft structures, specifically for strain analysis and noncontact flaw detection. Yet because it requires at least three consecutive images, it is more applicable for measuring static shape changes. Recently, researchers have focused on expanding the technique to capture deformations occurring over time. One such approach, the phase of difference method, ${ }^{2}$ takes one image of an object after deformation and several beforehand. We adopted this method to PSDSS, but noise reduced the image quality. For this system to be effective, new algorithms are needed to process the images.

To address these problems, we first integrated a liquid crystal variable retarder (LCVR) with a Wollaston prism (see Figure 1). ${ }^{3}$ The LCVR shifts interference patterns, while the prism is a shearing element. The prism causes light coming from two points on an object to interfere, creating a pair of superimposed images. We also developed a new approach, called the $(4,1)$ method, for capturing time-dependent deformation. Based on the phase of difference ${ }^{2}$ method, our technique takes four images before a specimen deforms and only one afterwards. In addition, we integrated multiple algorithms to improve image quality. We combined the sine-cosine (sin-cos) average filtering ${ }^{4}$ and multigrid unweighted least squares (MULS) unwrapping algorithm ${ }^{5}$ to extract deformation data from the image. We applied the basic optical setup and measurement theory of PSDSS ${ }^{1}$ to the LCVR and Wollaston prism compact unit. When the object's surface is perpendicular with the viewing direction, the surface slope $\frac{\partial w}{\partial x}$ is linearly related to the phase change, $\Delta \phi$, of the shearograms as:

$$
\frac{\partial w}{\partial x}=\frac{\lambda}{4 \pi \delta_{x}} \Delta \phi
$$

where $\lambda$ is the wave length and $\delta_{x}$ is the known amount of image shearing.

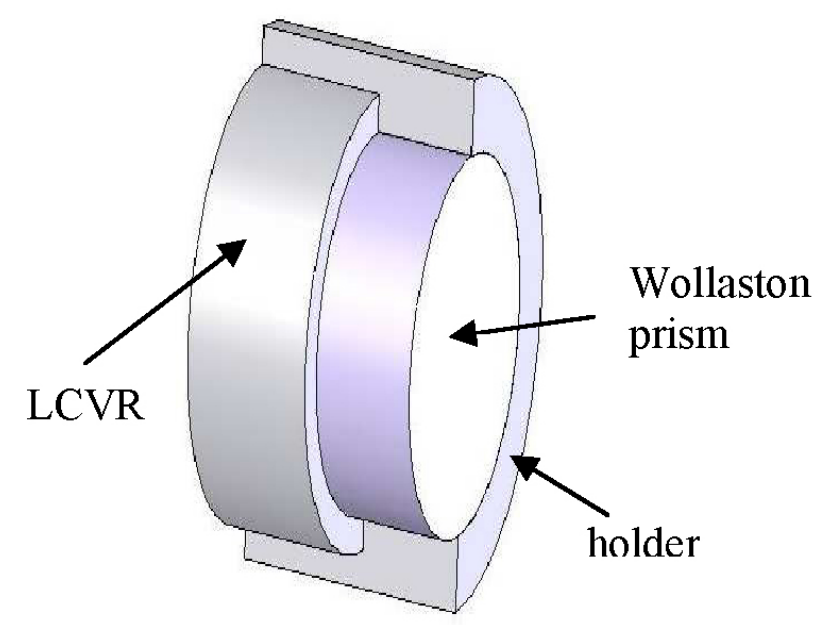

Figure 1. The combined liquid crystal variable retarder (LCVR) and Wollaston prism unit can provide accurate phase shifts.

We calibrated the LCVR to make sure it could provide precise phase shifts. Our group first adjusted the drive voltage of the LCVR continuously, which shifted the live shearographic fringes. Next, we simultaneously recorded these voltage variations and the gray value of a selected pixel on the shearogram. Finally, we plotted both variables and fit the data using a sinusoidal curve. This curve indicates the voltage required for a phase shift.

Next, we measured the out-of-plane surface displacement of a boundary-clamped plate. We applied a static, centered force to the plate by rotating the micrometer fixed to the back of the plate. We then measured its displacement quantitatively. By adjusting the LCVR, we captured four consecutive shearograms with a $\pi / 2$ phase shift. Figure 2 shows the sin-cos average filtered phase map and the final out-of-plane displacement. The results reveal the effectiveness of the LCVR and Wollaston prism unit, as well as the accuracy of the in situ calibration method. 


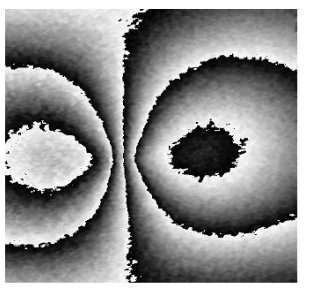

(a)

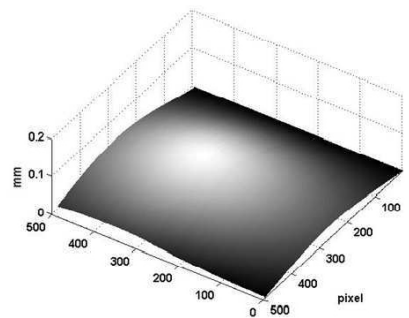

(b)
Figure 2. (a) Sin-cos average filtering improves the filtered wrapped phase map image quality. (b) The out-of-plane displacement map.

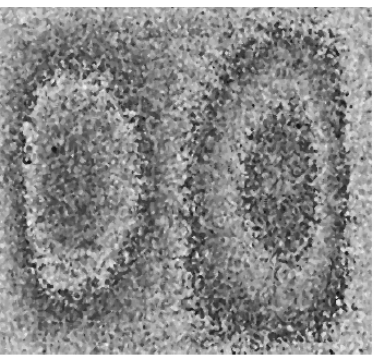

(a)

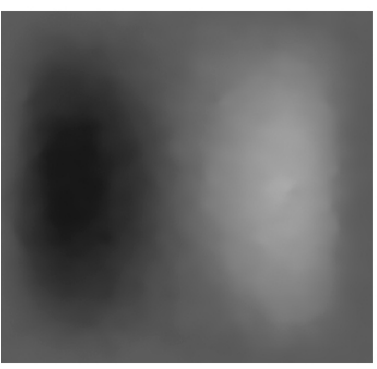

(c)

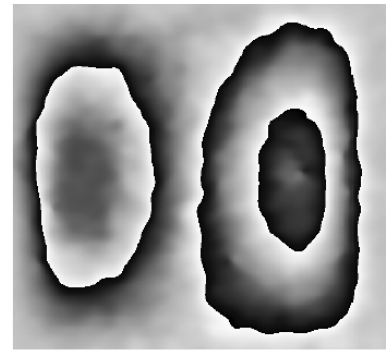

(b)

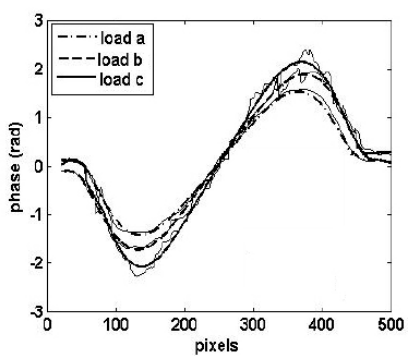

(d)
Figure 3. (a) Wrapped phase map. (b) Filtered wrapped phase map. (c) Unwrapped phase map. (d) The phase variations along the horizontal center line for three load levels can be used to calculate the slope and displacement.

For time-dependent applications, we relied on the $(4,1)$ method. We first measured the rectangular inner bonding defect on a composite plate. Next, we used the sin-cos average filter method on the the noise-corrupted wrapped phase map. We then unwrapped it using the MULS unwrapping algorithm (see Figure 3). We measured the object's deformation at three different load levels. Figure 3(d) shows the final calculated phase value along the horizontal center line. The slope and displacement can then be found from the fitted curves.
Our PSDSS compact system yields higher-quality images than existing approaches and holds promise for measuring timedependent shape changes. In addition, advanced processing algorithms effectively handled the noise. However, the current method can only measure small deformations. Further research will use new algorithms to tackle larger deformations.

\section{Author Information}

\section{Cuiru Sun and Hua Lu}

Ryerson University

Toronto, Canada

Cuiru Sun is pursuing a $\mathrm{PhD}$ at Ryerson University in Canada. Her research interests include 3D stress analysis using computer vision and fringe analysis. She is also interested in digital image processing and its application to several research areas, including electronic packaging.

\section{Jinlong Chen}

Tianjin University

Tianjin, China

Jinlong Chen is a professor of mechanical engineering at Tianjin University in China. His current areas of research are advanced experimental mechanics, biomechanics, mechanical behaviors of novel materials, and nondestructive testing.

\section{References}

1. W. Steinchen and L. Yang, Digital Shearography: Theory and Application of Digital Speckle Pattern Shearing Interferometry, Optical Engineering Press, SPIE, PM100, Washington, DC, 2003.

2. C. Kao, G. Yeh, S. Lee, C. Lee, C. Yang, and K. Wu, Phase-shifting algorithms for electronic speckle pattern interferometry, Appl. Opt. 1 (1), pp. 46-54, 2002.

3. J. Chen, C. Sun, X. Ji, and Y. Qin, Damage evaluation of subsurface defect in sandwich by phase-shifting digital shearography, Key Eng. Mater. 306-308 (1), pp. 399-404, 2006. 4. H. Aebischer and S. Waldner, A simple and effective method for filtering speckleinterferometric phase fringe patterns, Opt. Commun. 162, pp. 205-210, 1999.

5. D. Ghiglia and M. Pritt, Two-Dimensional Phase Unwrapping: Theory, Algorithms, and Software, Wiley-Interscience, New York, 1998. 